\title{
Assessing the Intraoperative Risk of Esophageal Perforation during Anterior Cervical Spine Surgery: A Study Using Intraoperative Computed Tomography
}

\author{
Atsushi Nakano, Yoshiharu Nakaya, Takashi Fujishiro, Sachio Hayama, Takuya Obo, Ichiro Baba and Masashi Neo
}

Department of Orthopedic Surgery, Osaka Medical College, Osaka, Japan

\begin{abstract}
:
Introduction: Using intraoperative computed tomography (iCT), we aimed to clarify the course of the esophagus and pharynx during anterior cervical spine surgery to estimate the risk of intraoperative injury.

Methods: Sixteen patients who underwent anterior cervical spine surgery with intraoperative CT for registration of a navigation system without release of blade retraction were included. To investigate the status of the retracted esophagus and pharynx, the distance between the nasogastric tube and center of the vertebra (NVD) was measured at each disc and vertebral level (C4-7) using axial CT. The location of the cricoid cartilage, which may affect the shift of the esophagus and pharynx, was noted. Presence or absence of contact between the esophagus and the edge of the surgical blade was investigated.

Results: The NVDs were 28.0, 28.3, 28.9, 27.2, 24.7, 19.9, and $13.8 \mathrm{~mm}$ at C4, C4/5, C5, C5/6, C6, C6/7, and C7, respectively; NVDs at C6/7 or more caudal levels were significantly shorter than those at C6 or more cranial levels $(\mathrm{P}<$ 0.001). The cricoid cartilage was observed at the C4-C5/6 level. Esophageal contact with the edge of the blade was observed in nine cases at C6 or more caudal levels.

Conclusions: The esophagus, which was placed at C6 or more caudal levels, was directly retracted by the blade. Nevertheless, the pharynx, which was placed at C6 or more cranial levels, was mostly retracted with the cricoid cartilage. Thus, the risk of direct esophageal injury was higher at C6 or more caudal levels than at cranial levels.
\end{abstract}

Keywords:

esophageal perforation, intraoperative CT, anterior cervical spine surgery, hybrid operation room, navigation surgery, complication

Spine Surg Relat Res 2020; 4(2): 124-129

dx.doi.org/10.22603/ssrr.2019-0026

\section{Introduction}

An esophageal perforation is one of the well-known complications of anterior cervical spine surgery. Although very rare, an esophageal perforation can cause dysphagia, superficial soft-tissue infection, deep infection with hardware failure, pseudarthrosis with osteomyelitis/discitis, and, in worse cases, infectious mediastinitis with sepsis and death. The incidence of these complications ranges from $0 \%$ to $1.62 \%{ }^{1-9)}$. Apart from a systematic review and two multicenter studies, published articles on esophageal perforation are mostly composed of case reports and small series ${ }^{10-12}$. Generally, in anterior cervical spine surgery, the esophagus and trachea are laterally retracted using surgical blades. Despite its rarity, the intraoperative position of the esophagus should be iden- tified after placing the surgical blades to avoid intraoperative esophageal injury and secure the surgical field. However, detailed radiographic or anatomical studies investigating the position of the esophagus during anterior cervical spine surgery remain scarce.

In recent years, research on the effectiveness of the intraoperative computed tomography (iCT) imaging system has been conducted. Thus, our institution introduced a hybrid operation room in April 2016. We can evaluate the correct bone removal and adequate implant placement during anterior cervical surgery using the navigation system. Furthermore, iCT images enable us to visualize not only bone structures but also, medical tubes, and surgical retractors, which incidentally demonstrate the status of the esophagus during surgery. The objective of this study was to investigate

Corresponding author: Atsushi Nakano, ort095@osaka-med.ac.jp

Received: April 15, 2019, Accepted: July 23, 2019, Advance Publication: September 4, 2019

Copyright (C) 2020 The Japanese Society for Spine Surgery and Related Research 
Table 1. Clinical and Intraoperative Computed Tomography Data for Each Patient.

\begin{tabular}{rccccccccc}
\hline Case & Sex & Age & Diagnosis & Method & $\begin{array}{c}\text { Surgical } \\
\text { level }\end{array}$ & $\begin{array}{c}\text { Cranial cricoid } \\
\text { cartilage } \\
\text { position }\end{array}$ & $\begin{array}{c}\text { Caudal cricoid } \\
\text { cartilage } \\
\text { position }\end{array}$ & $\begin{array}{c}\text { NG tube } \\
\text { position }\end{array}$ & $\begin{array}{c}\text { Blade } \\
\text { contact to } \\
\text { esophagus }\end{array}$ \\
\hline 1 & M & 69 & CSR & ACDF & C5-6 & C4 caudal & C6cranial & Middle1/3 & C6caudal \\
2 & M & 69 & OPLL & ACDF & C4-6 & C4 middle & C5/6 & Middle1/3 & None \\
3 & M & 66 & CDH & ACF & C4-6 & C4 cranial & C5/6 & Middle1/3 & None \\
4 & F & 41 & CDH & ACDF & C6-7 & C4 middle & C5/6 & Middle1/3 & C7 \\
5 & F & 82 & OPLL & ACF & C4-6 & C4 cranial & C5caudal & Right $1 / 3$ & None \\
6 & M & 47 & CDH & ACF & C5-7 & C4 caudal & C6cranial & Middle1/3 & C7 \\
7 & F & 38 & CDH & ACDF & C5-6 & C4 middle & C5/6 & Middle1/3 & C6caudal \\
8 & M & 49 & OPLL & ACF & C5-7 & C4 middle & C6cranial & Middle1/3 & C7 \\
9 & F & 45 & CDH & ACDF & C5-6 & C4 cranial & C5middle & Middle1/3 & C7 \\
10 & M & 41 & OPLL & ACDF & C4-6 & C4 middle & C5/6 & Middle1/3 & None \\
11 & M & 59 & CDH & ACDF & C6-7 & C4 caudal & C6cranial & Right $1 / 3$ & C7 \\
12 & F & 38 & CDH & ACDF & C4-6 & C4 cranial & C5/6 & Middle1/3 & C6 \\
13 & F & 49 & OPLL & ACF & C3-7 & C3/4 & C5/6 & Middle1/3 & C5-6 \\
14 & F & 53 & OPLL & ACF & C3-6 & C4 cranial & C5/6 & Middle1/3 & None \\
15 & M & 51 & OPLL & ACF & C3-6 & C4 middle & C6middle & Right $1 / 3$ & None \\
16 & F & 47 & CDH & ADR & C5-6 & C4 middle & C5/6 & Middle1/3 & None \\
\hline
\end{tabular}

CSR cervical spondylotic radiculopathy, $O P L L$ ossification of posterior longitudinal ligament, $C D H$ cervical disc hernia, $A C D F$ anterior cervical discectomy and fusion, $A C F$ anterior corpectomy and fusion, $A D R$ artificial disc replacement $N G$ nasogastric

the radiological condition of the esophagus retracted using a surgical blade and to assess the regional risk of esophageal injury via morphological assessment using iCT. To the best of our knowledge, this is the first study to reveal the intraoperative condition of the surgical blade-retracted esophagus.

\section{Materials and Methods}

This retrospective study was approved by our institutional ethical review board. Sixteen patients underwent anterior cervical surgery with iCT between August 2016 and September 2018. Of the patients, eight were male, and the average age of all patients was 53 years (38-82). Anterior cervical surgery was performed in eight patients with a herniated cervical disc, in seven patients with ossification of the posterior longitudinal ligament, and in one patient with cervical spondylotic radiculopathy. The operative methods included anterior cervical discectomy and fusion (ACDF) in eight patients, anterior corpectomy with fusion (ACF) in seven patients, and artificial disc replacement (ADR) in one patient. Operated spinal levels were C3-6 in two patients, C3-7 in one, C4-6 in five, C5-6 in four (including one ADR), C5-7 in two, and C6-7 in two (Table 1).

\section{Protocol for surgery and $\mathrm{iCT}$}

A radiolucent operating table composed of carbon was used in all patients. The patients were placed with their head fixed using a MAYFIELD radiolucent device. The neck position was mildly protruded and neutral in rotation, which is advantageous for maintaining an accurate midline during drilling and implant placement. The surgical procedure was performed via a standard anterior approach with a left access. Carbon blades with $19 \mathrm{~mm}$ width (CASPAR Cervical Retractor PEEK System, Aesculap, Germany) were placed under the bilateral longus coli muscles, and the trachea and esophagus were retracted to the right side. After a corpectomy and/or discectomy to some extent, intraoperative CT was obtained using a 128-row multidetector CT unit with a self-moving helical CT scanner gantry (SOMATOM Definition AS + SLIDE, Siemens, Germany) for the registration of the navigation system to check the extent of corpectomy and/or the position of the implants. For the navigation, a carbon fiber reference frame was placed on the MAYFIELD device. All iCT scans were performed under apnea ventilation following pre-oxygenation to reduce motion artifacts. The series consisted of $1.0 \mathrm{~mm}$-thick CT sections, which were acquired in the helical mode and were reconstructed at $1.0 \mathrm{~mm}$ intervals. The acquisition parameters were $130 \mathrm{kV}$ and $400 \mathrm{~mA}$. After completion of the scan, the acquired image data were automatically transferred to the in-house DICOM system and the intraoperative image-guided platform (Brainlab Spinal Navigation version 3.0 and Brainlab Curve $^{\mathrm{TM}}$, Brainlab AG, Feldkirchen, Germany). Registration of the navigation was automatically performed on the basis of the captured iCT data, and the optimal corpectomy area and implant position can be immediately checked without radiation exposure from the $\mathrm{C}$-arm.

\section{Radiological assessment with iCT}

In the present study, a nasogastric (NG) tube, which was preoperatively placed at the induction of anesthesia, was used as a reference for the position of the esophagus and to investigate the position of the retracted esophagus and phar- 
ynx. The distance between the tube and center of the vertebra (NVD) was independently measured (by T. O.) at each vertebral and disc levels (C4-7) using axial CT (Fig. 1). All measurements were conducted using a digitizer connected to a computer. To investigate the influence of the operative spinal level, the population was divided into two groups: cases with procedures involving $\mathrm{C} 7(\mathrm{C} 7(+)$ group) and cases without procedures involving $\mathrm{C} 7$ (C7(-) group). Then, the NVDs were compared. Localization of the cricoid cartilage, which may affect the shift of the esophagus and pharynx, was examined using axial CT. The lumen of the esophagus and pharynx were visualized by adjusting the CT window condition, and the localization of the NG tube in the lumen was evaluated. Presence or absence of contact between the esophagus and the edge of the surgical blade was investigated.

\section{Statistical analysis}

Difference in NVD among spinal levels was assessed by

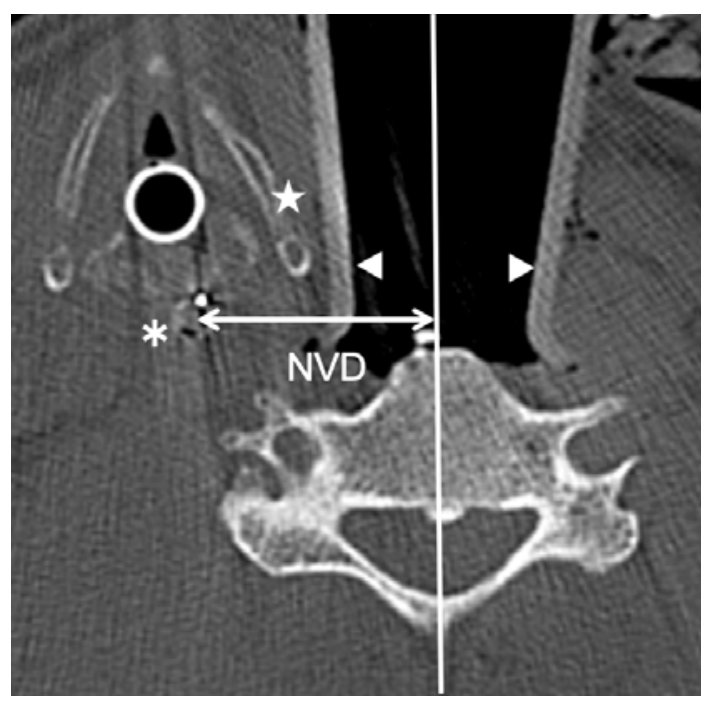

Figure 1. Intraoperative axial CT. To investigate the status of the retracted esophagus, the distance between the NG tube and the center of the vertebra (NVD) was measured at each vertebral and disc level. Asterisk indicates the NG tube. Star indicates the cricoid cartilage. Arrow heads indicate retractor blades. repeated measures analysis of variance followed by TukeyKramer Honest Significant Difference test for multiple comparisons. Comparison between the two groups $(\mathrm{C} 7(+)$ and $\mathrm{C}$ 7(-) groups) was performed using Wilcoxon rank sum test. A $P$ value $<0.05$ was considered statistically significant. All statistical analyses were performed using the JMP statistical package version 12.0 (SAS Institute Inc., Cary, NC, USA).

\section{Results}

Overall, the NVD was 28.0, 28.3, 28.9, 27.2, 24.7, 19.9, and $13.8 \mathrm{~mm}$ at $\mathrm{C} 4, \mathrm{C} 4 / 5, \mathrm{C} 5, \mathrm{C} 5 / 6, \mathrm{C} 6, \mathrm{C} 6 / 7$, and $\mathrm{C} 7$, respectively (Table 2). The NVDs at $\mathrm{C} 6 / 7$ and $\mathrm{C} 7$ were significantly shorter than those at C6 or more cranial levels $(P$ $<0.001$, Fig. 2A). No significant difference was observed at each level in the comparison of the two groups $(\mathrm{C} 7(+)$ group vs. C7(-) group) (Table 2). The NVDs of the C7(-) group at $\mathrm{C} 6 / 7$ and $\mathrm{C} 7$ were significantly shorter than those at C6 or more cranial levels $(P<0.001$, Fig. $2 \mathrm{~B})$; however, there was no significant difference in the NVD of the $\mathrm{C} 7(+)$ group at each level (Fig. 2C). With the exception of three cases, all NG tubes passed through the central third of the esophageal lumen. In three cases, the tubes passed through the right $1 / 3$ of the lumen at C6 or more cranial levels (Table 1). Contact between the esophagus and the edge of the blade was observed in nine cases $(56.2 \%)$ and at C6 or more caudal levels (Table 1$)$. In all cases, the cricoid cartilage was observed at the C4-C5/6 level (Table 1). The features of the surgical field were clearly visualized using reconstructed 3D CT images. All the NG tubes were sufficiently distant from the blade on the cranial side from C6, and they ran just beside the blade on the caudal side from $\mathrm{C}$ 6 (Fig. 3).

\section{Discussion}

As mentioned earlier, several studies revealed that the incidence of esophageal perforation during anterior cervical surgery is relatively low. Iatrogenic intraoperative injury during this surgical approach is a common cause of esophageal perforation; however, this represents only $19 \%$ of all perforations ${ }^{11}$. Contrarily, esophageal injuries are often obscure,

Table 2. NVD at Each Level and Frequency of the Esophageal Contact.

\begin{tabular}{|c|c|c|c|c|c|c|}
\hline & Level & Total $(\mathrm{N}=16)$ & $\mathrm{C} 7(+)$ group $(\mathrm{N}=5)$ & vs & $\mathrm{C} 7(-)$ group $(\mathrm{N}=11)$ & $\mathrm{P}$ \\
\hline \multirow[t]{7}{*}{$\mathrm{NVD}(\mathrm{mm})$} & $\mathrm{C} 4$ & $28 \pm 8.0$ & $22.0 \pm 7.1$ & & $30.2 \pm 7.3$ & 0.09 \\
\hline & $\mathrm{C} 4 / 5$ & $28.3 \pm 7.7$ & $23.2 \pm 8.2$ & & $30.2 \pm 7.0$ & 0.151 \\
\hline & $\mathrm{C} 5$ & $28.9 \pm 7.7$ & $23.9 \pm 8.6$ & & $30.7 \pm 6.9$ & 0.17 \\
\hline & $\mathrm{C} 5 / 6$ & $27.2 \pm 7.0$ & $25.6 \pm 9.1$ & & $27.9 \pm 6.2$ & 0.955 \\
\hline & C6 & $24.7 \pm 6.6$ & $24.3 \pm 10.2$ & & $24.9 \pm 4.8$ & 0.533 \\
\hline & $\mathrm{C} 6 / 7$ & $19.9 \pm 7.2$ & $21.7 \pm 11.0$ & & $19.1 \pm 5.2$ & 0.396 \\
\hline & $\mathrm{C} 7$ & $13.8 \pm 7.3$ & $17.0 \pm 10.4$ & & $12.3 \pm 5.4$ & 0.336 \\
\hline Contact between blade and esophagus & & $9 / 16(56 \%)$ & $5 / 5(100 \%)$ & & $4 / 11(38 \%)$ & 0.03 \\
\hline
\end{tabular}

$N V D$ distance between the nasogastric tube and the center of the vertebra 

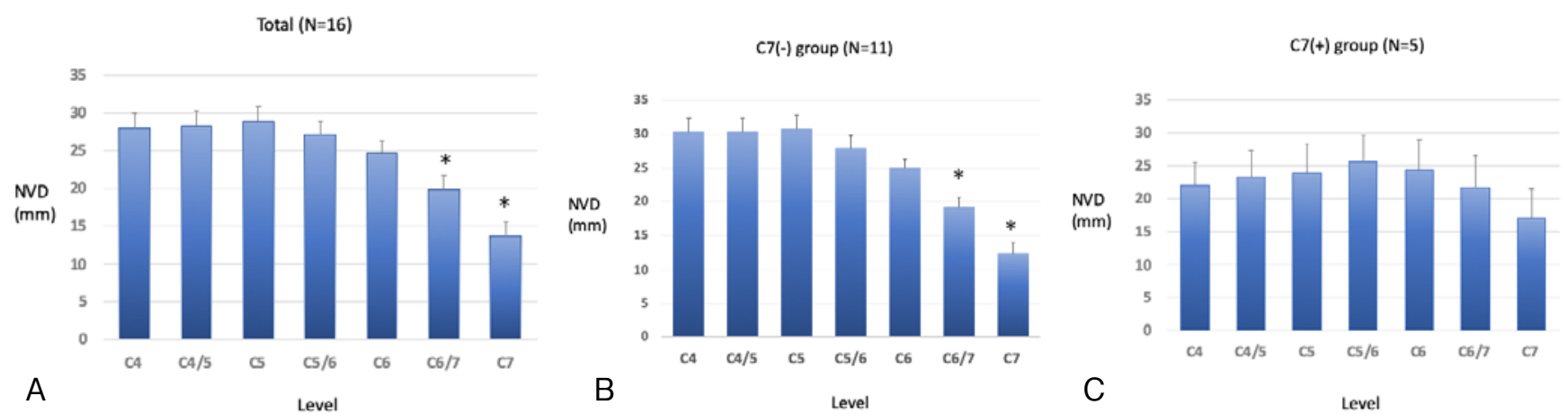

Figure 2. A: Distance between the NG tube and the center of the vertebra (NVD, mm) at each cervical level in total cases. NVD at $\mathrm{C} 6 / 7$ or more caudal levels was significantly shorter than that at C6 or more cranial levels (*). C7 vs. C4, C4/5, C5, C5/6, and C6; $P<0.01$. C7 vs. C6/7; $P=0.03$. C6/7 vs. C4, C4/5, C5, and C5/6; $P<0.01$. C6/7 vs. C6; $P=0.04$.

B: NVDs at each cervical level in the cases not involving C7. NVD at $\mathrm{C} 6 / 7$ or more caudal levels was significantly shorter than that at C6 or more cranial levels (*). C7 vs. C4, C4/5, C5, C5/6, and C6; $P<0.01$. C6/7 vs. C4, C4/5, and C5; $P=0.01$. C6/7 vs. C5/6; $P=0.04$.

$\mathrm{C}$ : No difference was observed among the NVDs at each level in the cases involving C7. NG tubes tended to be pulled evenly.

Error bar represent the standard error.
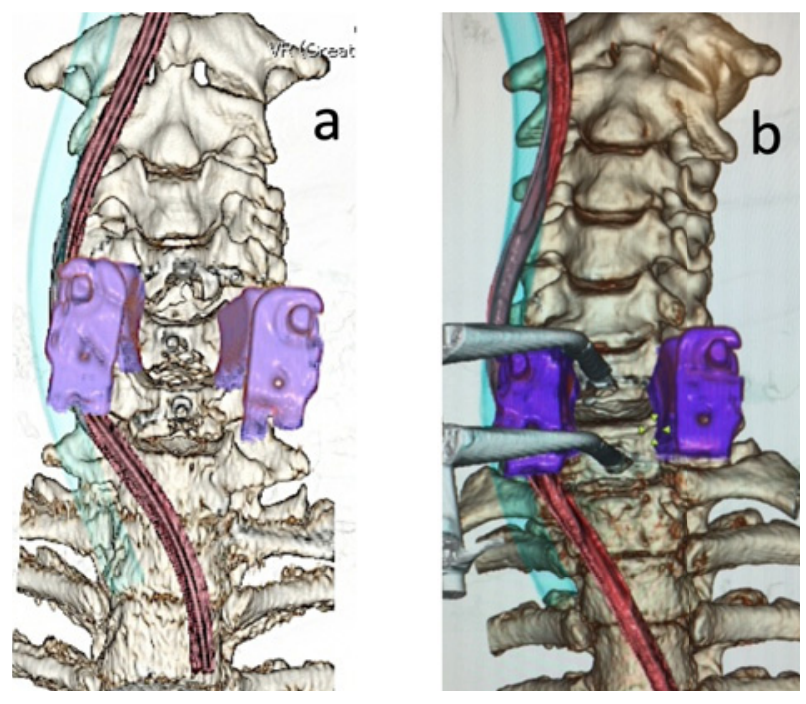

Figure 3. Reconstructed 3D CT. The red tube is a NG tube and the light blue tube is a tracheal tube. The NG tube is sufficiently distant from the blade on the cranial side from C6, whereas it runs just beside the blade on the caudal side from C6.

a: C4-6 ACF (case 11), b: C6/7 ACDF (case 12).

which makes them difficult to detect, especially when they are isolated ${ }^{13)}$. Thus, it is possible that esophageal perforation may be more frequent than is currently known. Inadvertent contact with a knife, high-speed burr, and misuse of electrocautery has also been cited as potential sources of direct intraoperative esophageal injury. Caution during the initial esophageal exposure, including adequate retractor blade placement under the longus colli muscle, has been suggested to minimize esophageal injury ${ }^{14,15}$. However, inadvertent esophageal "escape," which represents the protrusion of the esophagus into the operative field from under the retractor, sometimes cannot be avoided ${ }^{10)}$. To evaluate this phenomenon, we examined the anatomical and morphological details of the esophagus in the surgical field during anterior cervical surgery in the present study.

The pharynx is funnel shaped and extends from the cranial base to the inferior border of the cricoid cartilage anteriorly and the inferior border of the C6 vertebra posteriorly. The flat posterior wall of the pharynx lies against the prevertebral layer of the deep cervical fascia. The laryngopharynx, which is posterior to the larynx, is associated with the C4-6 vertebrae ${ }^{13)}$. In the current study, the cricoid cartilage, which affects the shift of the esophagus and pharynx, was observed at the $\mathrm{C} 4$ to $\mathrm{C} 5 / 6$ level. Because of this anatomical relation, most of the laryngopharynx (C4-6) is covered by the thyroid and cricoid cartilage anteriorly. Conversely, the esophagus is a muscular tube that is continuous with the laryngopharynx at the pharyngoesophageal junction. The cervical esophagus, which constitutes the upper third of the esophagus, slightly inclines to the left as it descends and enters the superior mediastinum, becoming the thoracic esophagus. The cervical esophagus immediately begins at the level of the inferior border of the cricoid cartilage in the median plane (at the level of the C6 vertebra), lies in front of the cervicothoracic vertebral junction, and enters the mediastinum ${ }^{13}$. Unlike the pharynx, the cervical esophagus is not covered with cartilages.

The current study is the first to visually reveal the intraoperative course of the esophagus retracted with a surgical blade. The NVDs at C6/7 and C7 were significantly shorter compared with those at $\mathrm{C} 6$ or more cranial levels. Contact between the esophagus and the edge of the blade was observed in nine cases alone, at C6 or more caudal levels. Thus, the pharynx, which was placed at C6 or more cranial levels, was mostly retracted with the cricoid cartilage (Fig. 4a). Nevertheless, the esophagus, which was placed at C6 or more caudal levels, was directly retracted by the blade (Fig. 4b). Thus, the risk of inadvertent esophageal "escape" and direct injury should be higher at C6 or more caudal levels than at cranial levels. Hershman et al. revealed in their 

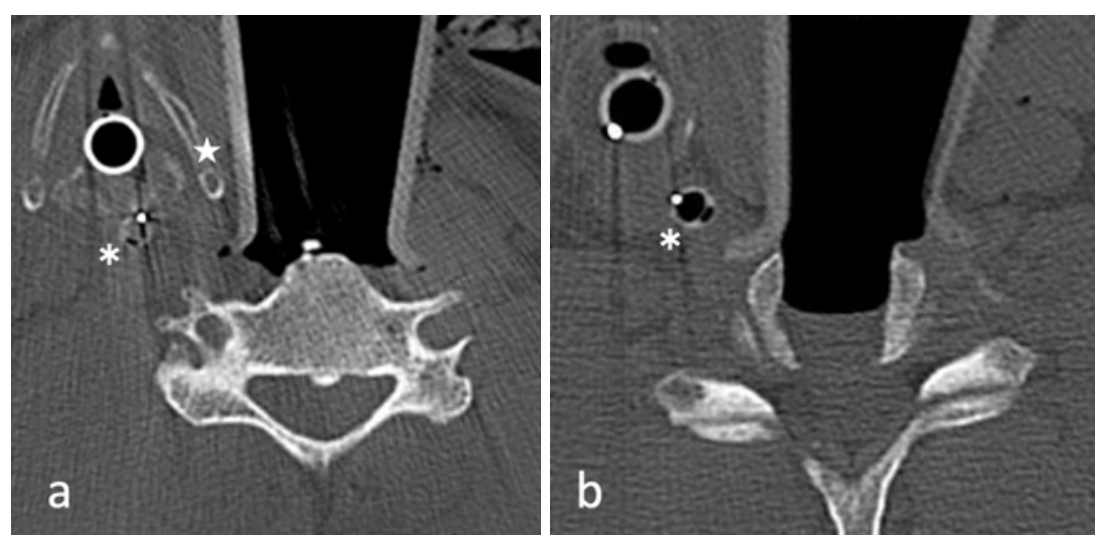

Figure 4. a: Axial CT scan at C5 level (case 10), b: Axial CT scan at C6/7 level (case 8).

The pharynx, which was placed at the C6 level or more cranial levels, was mostly retracted with the cricoid cartilage (Figure 4a). Nevertheless, the esophagus, which was placed at the C6 level or more caudal levels, was directly retracted by the blade (Figure 4b). Asterisk indicates the NG tube. Star indicates the cricoid cartilage.

review that the prevalence of esophageal injury at C5-7 was $68 \%-100 \%{ }^{10,12,16-18)}$. They highlighted that the portion of the esophagus that was most vulnerable to injury is known as Killian's triangle, which is formed by the junction of the paired inferior pharyngeal constrictor and cricopharyngeus muscles. This region, which usually lies anterior to the $\mathrm{C} 5 / \mathrm{C}$ 6 disc but is occasionally found more caudally, is particularly susceptible to injury as the posterior esophageal mucosa lacks muscular protection ${ }^{10)}$. In the current study, the NVD in the two groups was not significantly different if the procedure involved C7 (Table 2). In addition, when comparing NVD at each level within the $\mathrm{C}(+)$ group, no difference was noted and the NG tubes tended to be evenly pulled (Fig. 2C). In all cases involving C7, the contact between the esophagus and blade was observed at the $\mathrm{C} 7$ level. Conversely, contact with the esophagus was observed in $36 \%$ (4/ 11) of the C7(-) group (Table 1). Considering the results of the present study and several previous reports, we suggest that more care should be taken at the low cervical levels during surgery to reduce the risk of esophageal injury.

The present study has several limitations. First, only a few subjects were involved, and the involved spinal levels were diverse. However, the esophagus was retracted in the same way at different spinal levels (Fig. 3), which may be a universal phenomenon owing to the special anatomical features of the esophagus and pharynx. This finding should not change if the number of cases is increased. Second, CT is inferior to magnetic resonance imaging of soft tissue masses. Therefore, the center of the esophagus was defined as the center of the NG tube in the current study, which may not be accurate. We confirmed that all the tubes, except in three cases, were located at almost the center of the esophagus and pharynx by adjusting the CT value. Thus, NVD can be considered a reliable measurement for the study of iCT. Third, there were no cases in which the upper thoracic spine was involved. At the transition area of the cervical and thoracic spine, the intraoperative retraction of the esophagus is affected by the anatomical constriction due to the arch of the aorta. Although the results of the present study may not change, cases involving the upper thoracic spine should be examined in a future study.

In conclusion, the esophagus, which was placed at C6 or more caudal levels, was directly retracted by the blade. However, the pharynx, which was placed at C6 or more cranial levels, was mostly retracted with the cricoid cartilage. Consequently, the risk of direct esophageal injury was higher at C6 or more caudal levels than at cranial levels. Thus, we suggest that surgeons should consider the risk of direct intraoperative injury to the esophagus at C6 or more caudal levels when performing anterior cervical surgery.

Disclaimer: Masashi Neo is one of the Editors of Spine Surgery and Related Research and on the journal's Editorial Committee. He was not involved in the editorial evaluation or decision to accept this article for publication at all.

Conflicts of Interest: The authors declare that there are no relevant conflicts of interest.

Acknowledgement: The authors are grateful to Hashikawa $\mathrm{T}$ for the $\mathrm{CT}$ reconstruction imaging technique. We would like to thank Lee SW for the expertise regarding the esophageal anatomy and Editage (www.editage.jp) for the review of English language.

Author Contributions: Atsushi Nakano wrote and prepared the manuscript, and all of the authors participated in the study design. All authors have read, reviewed, and approved the article.

\section{References}

1. Amhaz HH, Kuo R, Vaidya R, et al. Esophageal perforation fol- 
lowing cervical spine surgery: a review with considerations in airway management. Int J Crit Illn Inj Sci. 2013;3(4):276-8.

2. Ahn SH, Lee SH, Kim ES, et al. Successful repair of esophageal perforation after anterior cervical fusion for cervical spine fracture. J Clin Neurosci. 2011;18(10):1374-80.

3. Dakwar E, Uribe JS, Padhya TA, et al. Management of delayed esophageal perforations after anterior cervical spinal surgery. J Neurosurg Spine. 2009;11(3):320-5.

4. Haku T, Okuda S, Kanematsu F, et al. Repair of cervical esophageal perforation using longus colli muscle flap: a case report of a patient with cervical spinal cord injury. Spine J. 2008;8(5):831-5.

5. Navarro R, Javahery R, Eismont F, et al. The role of the sternocleidomastoid muscle flap for esophageal fistula repair in anterior cervical spine surgery. Spine (Phila Pa 1976). 2005;30(20):E 617-22.

6. Phommachanh V, Patil YJ, McCaffrey TV, et al. Otolaryngologic management of delayed pharyngoesophageal perforation following anterior cervical spine surgery. Laryngoscope. 2010;120(5):930-6.

7. von Rahden BH, Stein HJ, Scherer MA. Late hypopharyngoesophageal perforation after cervical spine surgery: proposal of a therapeutic strategy. Eur Spine J. 2005;14(9):880-6.

8. Eleraky MA, Llanos C, Sonntag VK. Cervical corpectomy: report of 185 cases and review of the literature. J Neurosurg. 1999;90(1): 35-41.

9. Cloward RB. Complications of anterior cervical disk operation and their treatment. Surgery. 1971;69(2):175-82.

10. Hershman SH, Kunkle WA, Kelly MP. Esophageal perforation following anterior cervical spine surgery: case report and review of the literature. Global Spine J. 2017;7(1):28S-36.

11. Halani SH, Baum GR, Riley JP. Esophageal perforation after anterior cervical spine surgery: a systematic review of the literature. J Neurosurg Spine. 2016;25(3):285-91.

12. Newhouse KE, Lindsey RW, Clark CR, et al. Esophageal perforation following anterior cervical spine surgery. Spine (Phila Pa 1976). 1989;14(10):1051-3.

13. Moore KL, Dalley AF, Agur AM. Clinically oriented anatomy. 8th ed. Philadelphia: Lippincott Williams \& Wilkins; 2018. p. 1041-8.

14. Daniels AH, Riew KD, Yoo JU, et al. Adverse events associated with anterior cervical spine surgery. J Am Acad Orthop Surg. 2008;16(12):729-38.

15. Taylor B, Patel AA, Okubadejo GO, et al. Detection of esophageal perforation using intraesophageal dye injection. J Spinal Disord Tech. 2006;19(3):191-3.

16. Zhong Z, Jiang J, Qu D, et al. Esophageal perforation related to anterior cervical spinal surgery. J Clin Neurosci. 2013;20(10): $1402-5$.

17. Lu X, Guo Q, Ni B. Esophagus perforation complicating anterior cervical spine surgery. Eur Spine J. 2012;21(1):172-7.

18. Patel NP, Wolcott WP, Johnson JP, et al. Esophageal injury associated with anterior cervical spine surgery. Surg Neurol. 2008;69(1): 20-4.

Spine Surgery and Related Research is an Open Access journal distributed under the Creative Commons Attribution-NonCommercial-NoDerivatives 4.0 International License. To view the details of this license, please visit (https://creativeco mmons.org/licenses/by-nc-nd/4.0/). 